Editor, EDGAR W. WOOLARD

\title{
ON SOME PROPERTIES OF THE WATER-VAPOR SPECTRUM AND THEIR RELATIONS TO ATMOSPHERIC RADIATION
}

By W. M. Elgagser

[California Institute of Technology, Pasadena, Callf., July 1937]

Measurements of the absorption coefficients of water vapor in the far infrared region have been made by several investigators $(1,2,3)$. When Simpson first pointed out (4) that the transparency interval around wavelength $10 \mu$ is of fundamental importance for the heat balance of the atmosphere, a number of important results followed $(5,6)$. The existing data on the absorption coefficients are in rather pronounced disagreement with one another. In a recent paper by $\mathrm{H}$. Wexler (7), comparative curves are given which show that the values obtained by Hettner (1) in steam are almost everywhere much larger than the values of Weber and Randall (3) for moist air at room temperature. An explanation of this discrepancy will be given later. It will be shown that the values of Weber and Randall are probably rather close to the true values of the absorption coefficient in the lower layers of the atmosphere, whereas a decrease with decreasing pressure can be expected. The results of Fowle (2) exhibit particularly distinct deviations from Beer's law of proportionality between absorption coefficient and total mass of absorbing material present. These effects must be attributed to the character of the water vapor spectrum as a line spectrum and would not appear in a truly continuous absorption spectrum.

In the present paper we investigate the physical consequences of the fact that the water vapor spectrum in the far infrared consists of a large number of narrow lines. It will be shown that the average width of these lines is much smaller than intervals which could be resolved with the spectrographs used hitherto. For thick layers of vapor complete absorption is nevertheless reached in most parts of the spectrum. It can be shown that under such circumstances the total absorption is approximately proportional to the air pressure in the absorbing layers and is inversely proportional to the square root of the absolute temperature. There is an additional variation with temperature of a more complex type which cannot yet be quantitatively evaluated from the available mensurements, but it is probably not very large.

It seems certain that entirely reliable computations of the energy transfer through the atmosphere can only be made when more detailed measurements of the far infrared part of the water vapor spectrum are available. However, the results of the present paper may serve to apply reductions to measurements in order to account for pressure and temperature variations even if the measurements have not the full precision desired.

Before entering into our subject we might remark that in recent years considerable progress has been achieved in $26020-97-1$ our knowledge of the water molecule and the origin of its spectrum, mostly by the work of $R$. Mecke and his pupils (8). They have analyzed almost all of the water vapor bands in the near infrared, from the visible wavelengths up to the band at $6 \mu$. The farther infrared beyond $10 \mu$ has not yet been analyzed, principally because of the insufficient resolution of the measurements. For meteorological purposes in particular, the detailed analysis of the near infrared spectrum is of only minor importance; a detailed knowledge of the far infrared spectrum would be rather useful, as will be seen later.

\section{LINE STRUCTURE. PRESSURE AND TEMPERATURE DEPENDENCE}

The measurements, as well as the quantum theory of the water molecule, prove that the water vapor spectrum consists of a large number of very narrow spectral lines. We need a formula which gives the intensity distribution in a spectral line, $i$. e. the absorption coefficient as a function of the wavelength or frequency of the radiation. This distribution depends upon various conditions, such as pressure and temperature. A general theorem states that the total intensity, i. e. the absorption coefficient integrated over the line is a constant characteristic of the line. We therefore introduce a relativo intensity $i$ in such a way that the integrated intensity becomes unity. In order to obtain the true spectral absorption coefficient, we have only to multiply $i$ by the total intensity of the line. As is shown in radiation theory (9), the intensity distribution is given by the dispersion formula (see figure):

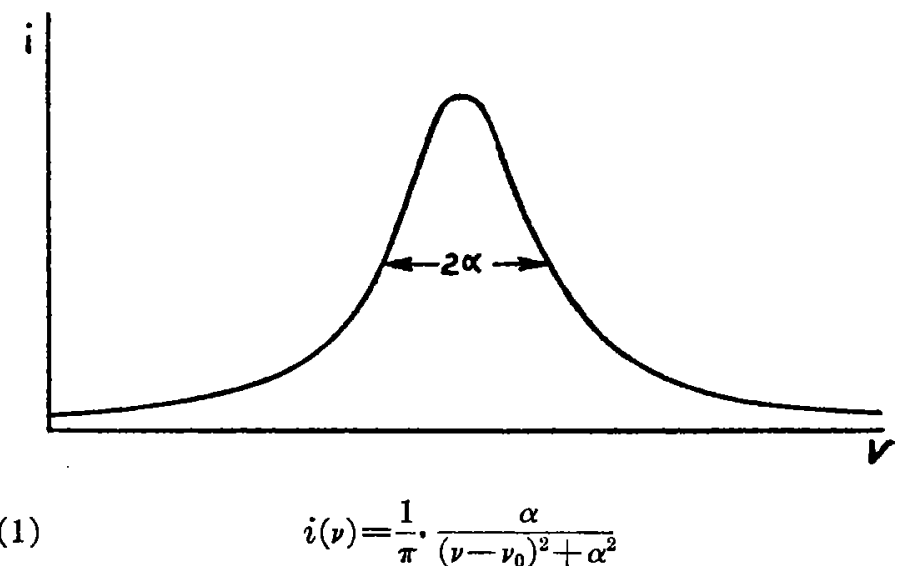

where $\nu$ is the frequency, $\nu_{0}$ the frequency in the middle of the line and $\alpha$ a constant. The dependence of the line 
shape upon physical conditions will appear in a variation of $\alpha$. One verifies readily that $\int i d \nu=1$. The limits of integration should be 0 and $\infty$, but since the integrand is already extremely small at $\nu=0$, one may extend the integration from $-\infty$ to $+\infty$. The maximum intensity which obtains at the line center is evidently

$$
i\left(\nu_{0}\right)=\frac{1}{\pi \alpha}
$$

For $\nu-\nu_{0}= \pm \alpha$ the intensity is half of the maximum intensity (2); therefore the quantity $2 \alpha$ represents the socalled half-width of the line in frequency measure. At great distances from the line center on the other hand, we may simplify (1) to

$$
i(\nu)=\frac{1}{\pi} \cdot \frac{\alpha}{\left(\nu-\nu_{0}\right)^{2}}
$$

The intensity is then smaller by a factor $\alpha^{2} /\left(\nu-\nu_{0}\right)^{2}$ than the maximum value (2). These parts of the spectral line will be called the "edges" of the line, whereas the part where the intensity is comparable to the maximum intensity will be called the "core" of the line. A spectral line has in principle an infinite extension; in a spectrum with many lines we shall only consider the absorption of one line up to a point where the absorption due to a subsequent line becomes larger than that due to the line in question. From (2) and (3) we see that the narrower a line-i. e., the smaller $\alpha$-the larger is the intensity in the core and the smaller the intensity in the edges.

We have now to find out what determines the factor $\alpha$. Radiation theory shows that a spectral line is never infinitely sharp, but the broadening of the lines due to electromagnetic causes is usually excessively small. Under the conditions realized in the atmosphere, line broadening is almost exclusively due to the perturbation of the radiating molecule by collisions with other gas molecules (10). As was first shown by $\mathrm{H}$. A. Lorentz (11), the molecular impacts interrupt the coherence of the wave train emitted by the radiating system and thus give rise to a broadening of the line. A more rigorous theory has been developed by Dennison (12) which will, however, not be needed for our present purpose, since the results do not differ greatly from those of the more elementary Lorentz theory. 'The constant $\alpha$ is found to be equal to

$$
\alpha=\frac{n}{2 \pi}
$$

where $n$ is the number of effective impacts per unit time. This result needs some comment. In the kinetic theory of gases one assumes the molecules to be rigid spheres. This, of course, is only a rough approximation and different methods for the experimental determination of $n$ give slightly different values. Many experiments have been performed to determine the broadening of lines in the visible spectrum (10). The effective number of impacts appears to be slightly larger than that yielded by the kinetic theory of gases. The latter (13) gives the value:

$$
\alpha=N_{0} \sigma^{2} p \sqrt{\frac{1+m_{w o} / m_{a}}{2 \pi m_{w o} R T}}
$$

where $N_{0}$ is Avogadro's number, $m_{w}$ and $m_{a}$ the molecular weights of water and air respectively, $R$ the gas constant, and $\sigma$ the effective diameter for an impact between a water and an air molecule. This formula is derived under the assumption that the partial pressure of water vapor is small compared to the air pressure. The value of $\sigma$ is not known with great precision; we may put to sufficient approximation $\sigma=3 \cdot 10^{-8} \mathrm{~cm}$. Inserting numerical values in (5), we have

$$
\alpha=1.4 \times 10^{7} \frac{p}{\sqrt{T}}
$$

where $p$ is expressed in millibars and $T$ in degrees absolute. From this we can easily calculate the half-width of the lines in the wavelength scale. Since $\lambda=c / \nu$, we have $\Delta \lambda=\Delta \nu \cdot \lambda^{2} / c$. Now taking $\Delta \nu=2 \alpha$ we obtain for the halfwidth at standard temperature and pressure

$$
\Delta \lambda=5.8 \times 10^{-4}\left(\frac{\lambda}{10}\right)^{2}
$$

where $\lambda$ and $\Delta \lambda$ are expressed in $\mu$. This formula shows that the half-width in the spectral interval which interests us is extremely small. In the measurements of Weber and Randall (3), for instance, the slit width of the spectrograph corresponds to an interval of about $0.04 \mu$ around a wavelength of $10 \mu$ and to a larger interval for greater wavelength. Although the value (7) may deviate slightly from the true width, due to the uncertainty in the molecular diameter $\sigma$, it results from an inspection of Weber and Randall's curves that the line width is small compared with the average distance of the lines. In the spectral interval between $12 \mu$ and $22 \mu$ the experimental curves show about 50 distinctly perceptible lines, thus an average line distance of $0.2 \mu$. For the absorption band around $6 \mu$ the measurements of Plyler and Sleator (14) indicate a number of about 60 lines in the spectral interval from $5.7 \mu$ to $6.7 \mu$, whereas formula (7) yields a line width of only $2.10^{-4} \mu$. The experiments show that with the exception of the gap around $10 \mu$ the absorption in sufficiently thick layers of water vapor is practically continuous. We may therefore conclude that the main part of the absorption in thick layers takes place in the edges of the lines. Since according to (3) the absorption coefficient decreases as the inverse square of the distance from the line center, the absorption in the core must be very intense. In thin layers the absorption will take place in the cores only, and since complete absorption there will already be reached in a very thin layer, we may expect for a certain interval of thickness to have absorption of a definite fraction of the primary radiation, this fraction varying only slowly with thickness. This is just what was observed by Fowle (2) for a path of about $100 \mathrm{~m}$ in atmospheric air and for the spectral region beyond $10 \mu$. For computation of atmospheric transmission on the other hand one usually divides the atmosphere into layers of $1 \mathrm{~km}$ thickness and for such thicknesses the absorption may approximately be treated as semicontinuous in the manner of Simpson. We consider now especially this case. We divide the whole of the spectrum where continuous absorption takes place in a sum of intervals belonging to the line cores and in remaining intervals belonging to the line edges. We may for instance define as "core" everything that lies within a distance of $2 \alpha$ from the line center. Since within the cores the absorption coefficient is very large, a "black" radiation flux will be established within these intervals. Now it is well known that, for a black atmosphere, the radiative transmission does not depend upon the absolute value of the absorption coefficient, but only upon the temperature gradient $d T / d z$. We have assumed that our layer is so thick that complete absorption takes place even in the edges of the lines. Therefore the radiative transmission of our layer per unit spectral interval will be of the same order of magnitude for the core intervals as for the edge intervals. Since the core intervals cover only a very 
small fraction of the spectrum, we may, in a first approximation, neglect altogether the transmission in the cores. The absorption in the edges is by (3) proportional to $\alpha$ and according to (5) or (6) this means that the absorption coefficient is proportional to the air pressure and inversely proportional to the square root of the absolute temperature.

It is evident from the way this result has been derived that it should not be used for calculating the fraction of light absorbed in a moderately thin layer. Such a calculation would yield a much more complicated function. In computations of atmospheric radiation we are interested in certain critical thicknesses; for instance all layers of smaller thickness than a critical one will be considered as transparent and all layers larger than a critical thickness will be considered as black. Other circumstances being the same, the critical thickness is proportional to the air pressure.

This result cannot be checked by the available measurements on water vapor. However Kussmann (15) has performed measurements on the infrared spectrum of $\mathrm{HCl}$ in the spectral region around $100 \mu$; since the $\mathrm{HCl}$ and the $\mathrm{H}_{2} \mathrm{O}$ molecules have a closely analogous structure, these results might partly also apply to water vapor. Kussmann investigates the absorption for various partial pressures of $\mathrm{HCl}$ in the presence of various foreign gases $\left(\mathrm{CO}_{2}, \mathrm{~N}_{2}, \mathrm{~A}, \mathrm{H}_{2}\right)$. $\mathrm{He}$ finds that for a given partial pressure of $\mathrm{HCl}$ his results can be representied by assuming the absorption coefficient as a linear function $a+b p$ of the pressure $p$ of the foreign gas. The results of Kussmann cannot be interpreted in a very simple way. According to formula (6) the line width is much larger in this spectral region and this is only partly compensated by the reduced pressure. It is possible that the effect of the cores cannot be neglected in this case. The dependence upon the pressure of the foreign gas is the stronger the smaller the partial pressure of the HCl. If we are allowed to extrapolate for a very small partial pressure of $\mathrm{HCl}$, we obtain approximate proportionality of the absorption coefficient with total pressure in a rather large pressure interval. It is, however, doubtful whether this corresponds to the conditions of water vapor in the atmosphere, since nothing is known about the degree of absorption in the cores for $\mathrm{HCl}$.

\section{O'IHER EFHEC'S}

While the results of the preceding section can easily be applied to reduce the absorption coefficient according to changes in pressure and temperature, there exist a number of minor effects of a more complicated nature which shall be briefly reviewed here.

A first question concerns efiects of line broadening other than the one discussed above $(\mathbf{1 0})$. One cause of line broadening is to be found in the fact that the molecules are in movement while emitting light; this results in a shift in frequency of the emitted light (Doppler shift). In the average over all velocities and directions of the molecules a brondening of the line appears. This effect is iudependent of the pressure and directly proportional to the square root of the absolute temperature. A numerical calculation shows that for atmospheric temperatures the line width thus generated is equal to the width (6) if the latter is taken for a pressure of about one-tenth atmosphere. Thus in the troposphere the considered effect is negligible, while in the higher levels of the strntosphere the formulae of the previous section become invalid.

Formula (5) refers to a case where there are many more air than water molecules. For an atmosphere of pure steam, the constant $m_{A}$ would have to be replaced by $m_{W}$.
Experiments show that in this case a stronger broadening is to be expected, i. e. molecules of the same kind produce a larger perturbation of a radiating molecule than molecules of a different kind. This effect is very general (10). It can be taken into account in formula (5) by assuming a larger effective diameter $\sigma$ for an impact between two water molecules than for one between a water and an air molecule. This effect combined with the temperature dependence of the total line intensities to be considered in a moment is the cause of the larger absorption coefficients observed by Hettner (1) in steam as compared with Weber and Randall's values in moist air (see 7). It is therefore not advisable to use absorption coefficients of steam for computations of atmospheric radiation, the errors introduced by them being quite appreciable.

H. Becker (16) has measured directly the width of absorption lines of $\mathrm{HCl}$ in the near infrared at $1.7 \mu$. His values for the half width are about ten times larger than those calculated by formula (7). By the reasons just explained and by some others which we cannot give in detail here, it may rather definitely be assumed that the line widths for water under atmospheric conditions are smaller and much closer to the values derived from the kinetic theory of gases. The considerations of the previous section would of course remain valid even with considerably increased line width, since the mutual distance of the lines is in most parts of the spectrum so mucb larger than the width.

All the effects discussed so far refer to a change in the shape of a spectral line, the total intensity of the line remaining unaltered. We must now ascertain whether the total line intensities can change according to conditions. It can be shown that pressure has no effect upon the integrated intensities, while temperature has to a certain degree. According to quantum theory an emission or absorption of radiation corresponds to a transition between two distinet energy levels in a molecule. Let $\epsilon$ be the energy of a molecular level; then the relative concentration of molecules being in this particular state is according to Boltzmann's principle proportional to $e^{-\epsilon / k T}$. We are only interested in the relutive change of the concentration with $T$. If the excitation energy $\epsilon$ is small, the Boltzmann function will be close to unity and a change in $T$ has only a slight effect upon it. However, if the excitation $\epsilon$ is large, then the relative concentration $e^{-\varepsilon / k T}$ of such states becomes small, but its variation with temperature becomes rather large. Returning now to the water molecule, a closer consideration of the structure shows that the spectral lines in the region between, say, $10 \mu$ and $20 \mu$ originate in higher excited states of the water molecule. Boltzmann's formula shows that a decrease in tempernture results always in a decrease of the concentration of these states and thus in a decrease of the corresponding absorption coefficient. We cannot, however, determine this effect quantitatively, until this part of the spectrum has been analyzed. All we can siy is that the absorption coefficient of water in the higher, colder parts of the atmosphere may in this spectral region be somewhat lower than the measured values. It seems difficult to decide at present how much of the difference in the absorption coefficients of steam and of moist air has to be attributed to this effect.

We consider now the $\mathrm{CO}_{2}$ absorption band around

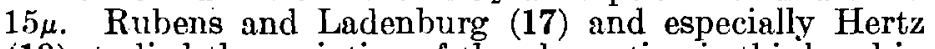
(18) studied the variation of the absorption in this band in dependence upon pressure and partial pressure. Although the variation is measurable, it is comparatively small Thus, for instance, the same amount of $\mathrm{CO}_{2}$ gives rise to an absorption of 86 percent in the middle of the band, if 
under a pressure of $40 \mathrm{~cm}$ mercury and to 70 percent absorption under a pressure of $9 \mathrm{~cm}$. This behavior is quite different from that of water vapor. It can be inferred from theoretical arguments that the individual lines which compose the absorption band of $\mathrm{CO}_{2}$ must be rather close together. One can prove that if the cores of the lines do completely overlap, the absorption becomes independent of pressura. In the $\mathrm{CO}_{2}$ band this condition is approximately realized. For computations of atmospheric transmission we may neglect the pressure dependence of the absorption in cases in which no great precision is required. There seem to be no other constituents of the atmosphere which absorb, appreciably, infrared radiation; ozone (19) in the concentrations in which it is present in the atmosphere is almost transparent in the far infrared.

\section{CONCLUSIONS}

In the preceding section we have tried to give an exhaustive enumeration of all the effects which under atmospheric conditions could possibly modify the absorption coefficient of water vapor. The main effect is the dependence upon pressure and temperature treated in the first section. There are two accessory features to be taken into account. The first is the appearance of gaps of partial or complete transparency in spectral regions which in Simpson's method are still treated as absorbing continuously; this refers especially to the region beyond $15 \mu$. The gaps will appear at places intermediate between two lines; these effects can be calculated from the formulae given above, when somewhat more precise values of the line intensities are known. The second effect is the change of the total line intensities due to the change in concentration of the corresponding molecular states.

All the effects which have been mentioned in this paper tend to decrease the absorption coefficient as compared with the values used so far, especially in the higher levels of the atmosphere. Our results lend themselves to a number of applications concerning the absorption of ter- restrial and solar radiation by the atmosphere which will be given at a later time. We might confine ourselves here to a few preliminary remarks. Simpson assumes in his work a content of about $0.3 \mathrm{~mm}$ of precipitable water for the whole of the stratosphere. Now under the reduced pressure this layer has an absorption corresponding to less than one-fifth of this amount of water under normal pressure. It follows that the stratosphere is practically transparent with the exception of the very narrow spectral regions occupied by the line cores. The flux of radiation through the stratosphere takes place principally in the cores; the radiation is therefore of a different spectral composition from that emitted by the troposphere. No direct inferences about the thermal state of the stratosphere can thus be drawn from a knowledge of the radiative transfer in the troposphere.

\section{REFERENCES}

(1) H. Rubens and G. Hettner, Verhandl. deut. phys. Ges. 18, 149 (1916); G. Hettner, Ann. der. Physik, 55, 476 (1918).

(2) F. E. Fowle, Smithsonian Misc. Coll. vol. 68, No. 8 (1917).

(3) L. R. Weber and H. M. Randall, Phys. Rev. 40, 835 (1932).

(4) G. C. Simpson, Mem. Roy. Meteor. Soc. vol. 3, No. 21 (1928).

(5) G. C. Simpson, Mem. Roy. Meteor. Soc. vol. 3, No. 23 (1929).

(6) C. G. Abbot, Smithsonian Misc. Coll. vol. 82, No. 3 (1929).

(5) H. Wexler, Monthly Weather Review, 64, 122 (1936).

(8) R. Mecke and others, Zeits. $f$. Physik, 81,313, 445 and 465 (1933).

(9) See e. g., Leigh Page, Introdnction to Theoretical Physics, Ch. XII.

(10) See e. g., A. Mitchell and M. Zemansky, ResonanceRadiation and excited Atoms.

(11) See V. Weisskopf, Physikal. Zeits. \$4, 1 (1933).

(12) D. M. Dennison, Phys. Rev. $\$ 1,503$ (1928).

(13) See e. g., Geiger-Scheel's Handbuch der Physik, vol. IX, p. 399

(14) E. K. Plyler and W. W. Sleator, Phys. Rev. S\%, 1493 (1931).

(15) H. Kussmann, Zeits. f. Phys. 48, 831 (1928).

(16) H. Becker, Zeits. f. Phys. 59,583 (1929).

(17) H. Rubens and F. Ladenburg, Verhandl. deut. phys. Ges. 7 , $170(1905)$.

(18) G. Hertz, Verh. deut. phys. Ges. 1s, 617 (1911).

(19) G. Hettner, R. Pohlmann and H. J. Schuhmachez, Zeits. f. Phys. 91, 371 (1934).

\title{
ON PILOT BALLOONS AND SOURCES OF LIGHT FOR HIGH ALTITUDE UPPER-WIND OBSERVATIONS
}

\author{
By William H. Wenstrom, Major, United States Army (retired)
}

[New Haven, Conn., October 1937]

The research described herein was begun in December 1934 , in response to military meteorological problems, and continued as occasional opportunity offered until September 1937 when the work was necessarily terminated on account of administrative reasons. Due to these limitations, some of the data obtained are incomplete, and the results presented should be regarded as first approximations. They are nevertheless thought to be of general value to meteorologists, because the field has previously been little explored.

When cloudiness or poor visibility exists in the lower levels, upper wind determinations to high altitudes can be made only by means of the yet experimental radio pilot balloon, ${ }^{1}$ or by means of expensive military techniques. The radio pilot-balloon problem is a complicated one, and its two-dimensional solution with a desirable precision of direction ( $1 / 10$ of 1 degree) will probably require many years. The research described herein is concerned only with the far more simple problem of determining upper winds during the day or night to altitudes of 15,000 to

\footnotetext{
1W. H. Wenstrom, Radiometeorography as Applied to Unmanned Balloons,
MoNTHLY WratHER RrVLEW, vol.62, July 1934.
}

40,000 feet in good visibility under clear skies or high cirriform clouds. Recent aviation trends toward flying in the substratosphere, 20,000 to 30,000 feet, have brought this problem into immediate practical importance. In addition, high-altitude upper-wind observations are often useful to a weather analyst and forecaster.

\section{PILOT BALLOONS}

Standard 6-inch pilot balloon.-The standard 6-inch pilot balloon, as used by the United States Weather Bureau and the Army Meteorological Service, weighs about $1 \mathrm{oz}$. $(30 \mathrm{~g})$ and costs about 10 cents. Inflated with hydrogen (cost about 10 cents) to a free lift of $4.66 \mathrm{oz}$. (137 g), corresponding to a sea-level diameter of about $21^{\prime}$ feet, it rises at an average rate (except in the zone of lower-level turbulence) of about 200 yards per minute. White, red, and black colors are available for use, respectively, under clear sky, cirriform clouds or darker clouds. A normally inflated balloon usually bursts at an altitude around 30,000 to 40,000 feet; but due to its small size, slow rise rate, increasing winds at upper levels, and the 